During the course of acute infections, especially influenza, meningeal symptoms may appear, due to "meningismus"that is to say, an increased formation of cerebro-spinal fluid without infection of the meninges. Professor Abram and others have shown that withdrawal of cerebro-spinal fluid by lumbar puncture rapidly relieves this condition. Wegeforth and Latha $\mathrm{m}^{3}$ objected to this procedure on the grounds that if a pneumococeal septicaemia be experimentally induced in rabbits, lumbar puncture increased the incidence of meningitis in animals so treated, apparently by drawing organisms towards the choroid plexus. Though the clinical opportunity has not yet arisen, it is obvious that hypertonic saline given intravenously would be free from this objection in the meningismus of acute infections; in fact, as Weed's later work ${ }^{4}$ shows, there would be a tendency for the organisms to be dragged away from the choroid plexus, for the current would be, not from blood to fluid as in the case of spinal puncture, but from fluid to blood.

\section{Cerebral Herniae.}

The first case on which we tried hypertonic saline was a girl who had been operated on, for a tumour of the cerebellopontine angle, by the subtentorial route. Subsequently a hernia as large as a Jaffa orange developed; it was associated with headache, vomiting, vertigo, and marked ataxia. Not only did hypertonic salines cause disappearance of the headache, but also marked improvement in the other symptoms, especially the gait; at the same time the size of the hernia decreased and it became much softer. This marked amelioration of the patient's condition continued for two months during her stay in hospital, an injection being given every third or fourth day. On discharge her symptoms recurred despite the fact that she was taking 15 grains of sodium chloride by the mouth thrice daily. Four cases of postoperative cerebral herniae have since been treated by intrarenons hypertonic salines, with gratifying results as far as srmptoms are concerned. It cannot be too strongly emphasized that the treatment is purely symptomatic in these cases, not curative.

\section{Hypertonic Saline prior to Operations for Cerebral} Tumour and Allied Conditions.

It has been shown that the lowering of cerebro-spinal fluid pressure resulting from the intravenous injection of hypertonic saline solutions is accompanied by a diminution in the volume of the brain. To those who have seen the bulging of brain substance through the bony cranial defect during a cerebral operation-a bulging which increases the surgeon's difficulties many times an, $i$ causes unnecessary damage to the brain substance-it is surprising that the pre-operative use of hypertonic salines is not more widely known.

\section{Other Uses.}

Further uses for these solutions readily suggest themselves. A woman, aged 28, was first seen eighteen months ago with one year's history of headache, occasional vomiting, and dystrophia adiposo genitalis. There were no signs of local pressure in the ineighbourhood of the pituitary fossa, and no change in the risual fields or fundus oculi. Injections of hypertanic salines failed to relieve her headache. During the last eighteen months no further evidence of a cerebral tumour has developed, and it can probably be excluded.

A girl, aged 18, with a similar Fröhlich's syndrome, but with secondary optic atrophy and definite evidence of a suprasellar tumour, obtained considerable relief from headache when given salt by the mouth.

Whether this points to the use of hypertonic salines in differentiating intracranial from extracranial causes of headache there is not yet sufficient evidence to show.

In cases of cerebral tumour, when cerebro-spinal fluid is desired for diagnostic purposes, but lumbar pnncture is feared lest "foraminal crowding" occur, it would seem that hypertonic salines given fifteen minhtes before puncture would minimize the risk.

Whilst hypertonic salines diminish both the volume of the brain and cerebro-spinal fluid pressure, hypotonic salines produce the opposite effect; they increase the volume of the brain and the cerebro-spinal fluid pressure. The practical value of this would seem to lie in the use of hypotonic salines in cases of headache occurring after lumbar puncture. In one case the headache completely subsided after the intravenous injection of hypotonic saline.
The respiratory effects-shallowness of respiration and partial failure - seen in experimental animals have not been observed in the cases here recorded. We have been unable to avail ourselves of the opportunity offered to investigate the rate of chloride excretion, the effect on urine output, alterations in blood volume, etc., following the injection of hypertonic salines. The few indications and results here recorded are sufficient to illustrate the uses and great value of this method in rational therapeutics.

\section{REPERENCES.}

1 Weed and McKibben : Amer. Journ. Physiol., 1919, xlviii, pp. 512. 531 2 Foley and Putman: Amer. Journ. Physiol., 1920, liii, p. 464 . 3 Wegeforth and Latham : Amer. Joum. Mell. Sci., 1919, clviii, p. 183. "Weed: Amer. Journ. Anatomy, 1923, xxxi, p. 191.

\section{SYSTEMIC POISONING BY HAIR DYE.}

\author{
BY \\ H. W. NOTT, M.R.C.S., L.R.C.P., \\ LITTLE SUTTON, BIRKENHEAD.
}

IT is well known that some phenylenediamine dyes cause irritation and dermatitis when conveyed to the skin of susceptible people by dyed furs, but hitherto I have not seen any reference to the serious consequences which may result to some from absorption of this poison.

The proprietor of a hairdressing saloon, of middle age, looking apparently well and who presented no physical signs of disease apparently well and who presented no physical signs of disease, came for examination on account of serious and unaccountable horrible sensation of sickness, weakness, and a feeling of impending death." He gave his story in a jerky, nervous manner and with an air of puzzled mystery, saying that he bad been subject to these seizures for nearly three years; that in the space of a few minutes he would pass from a feeling of good health into a state of pain, palpitation, and exhaustion; "he was going to die"; and on one pasion when he was preparing for bed an attack came so suddenly occasion when he was preparing for. bed an attack came so suddenly and with such tumultuous action of the heart that he fell on the
bed helpless, certain he was dying, and lapsed into unconsciousness, from which he must have passed into natural sleep, for he awoke next morning perfectly well, to" find himself lying across the bed half dressed. He said he had often been diagnosed at sight as a man "very ill with influenza," and had of ten been sent home to bed with his temnerature up. He had had several sojourns of from three to six weeks in bed getting over the "flu," but as a from three to six weeks in bed getting over the "flu,"

His story was substantiated by a large packet of prescriptions in which valerian, bromides, bismuth, and magnesia had been ordered. He had also received two courses of vaccines, and once had been threatened with removal to a sanatorium for tuberculous disease. As there seemed no reason to suspect alcoholism he was advised to watch whether any article of food might be poisoning him.

A week later he telephoned at 9 a.m. saying he had gone to bed perfectly well the night before and had wakened that morning to find himself in for another attack. When scen at 3 p.m. the same day he at first sight gave the impression of one who might have had several nights' drunken debauch, but his breath was perfectly healthy. His face was grey, cyanosed, and blotchy. His eyes were watery, the conjunctivae injected, and the evelids swollen. The lips and ears were violet, the tongue was swollen and covered with a thick yellow and brown fur. The gums were very swollen, standing away from the teeth, and purple. Some of his fingers were stained, away from the teeth, and purple. Some of his fingers were stained,
apparently with iodine.

strong; the temperature was normal.
He was nauseated, and had taken no food all day. He complained of pain, chiefly in the epigastric and hypochondriac regions, and between the shoulders. There was slight palpitation, and he looked anxious and strained. He described some difficulty in swallowing, with a burning sensation in the throat and behind the sternum. When he took a deep inspiration he felt severe pain in the chest, When he took a deep inspiration he felt severe pain in the chest, and a choking sensation. As the previous day, Sunday, had been home, it was impossible to resist thoughts of secret poisoning by arsenic, for he somewhat resembled a series of cases seen here twenty years ago when arsenic accidentally contaminated the beer twenty years ago when arsenic North of England.

When it was explained that his symptoms resembled some forms of poisoning, and when he began to think of the possibility of some of poisoning, and when he began to think of the possibility of some chance method of poisoning, he supplied the che himself by asking if hair dye could do it, for it was about three years ago when he
first used it. He often worked in the fumes of it, and occasionally applied the dye to his customers without wearing gloves, which accounted for the stains on his fingers-the last occasion being about forty-eight hours before this interview.

Two bottles of the dye were sent to an analyst with a note that it was under suspicion for causing mixed symptoms of lead and arsenic poisoning in a man who worked with it, and the following report was received :

" In aceordance with your instructions we have examined the two solutions of hair dye which you handed to us. 
"One solution gives the reactions for both metaphenylenediamine and paraphenylenediamine; the other is a solution of hydrogen peroxide.

"Paraphenylenediamine is commonly used as a hair dye, and injurious effects on workpeople engaged in the manufacture of this compound are attributed to the diamine itself and to its oxidation products.

"Metaphenylenediamine possesses marked poisonous properties, its physiological action resembling that of the leucomaines and ptomaines. An authority states that a dose of 0.1 gram per kilo body weight produced salivation, vomiting, diarrloea, and abundant excretion of urine in dogs and subsequent death by coma. Symptoms similar to those of intense influenza were also prodiced in addition similar to those of intense

" the more severe effects.

"Phenylenediamine derivatives are used in the production of dyes for furs and are commonly used in the preparation of brown dyes."

Three months have elapsed since the report was received, and by avoiding exposure to further risk of absorption, the patient has had no return of his illness.

The case adds further evidence of the peculiarly selective action of phenylenediamine, and seems to suggest the existence of a class of people who are extremely susceptible to its ill effects and who need not necessarily show any lesions of the skin. This is important, for bottles of this dye are issued in boxes containing notices warning people to test its effect on a small area of skin before using it, and are advised not to use it at all if a reddening of the skin results some hours after the experiment is made.

The action of the dye as a systemic poison may be delayed, for in this case a day and a half intervened between the last exposure and the onset of symptoms; as it was due to this delayed action that he had never associated his symptoms with his occupation, the question arises whether others may not be upsetting their health to a greater or less extent by dyeing their hair and moustaches.

It would appear from recent literature* on the subject of fur dermatitis that the toxic phenylenediamine becomes nontoxic when oxidized by hydrogen peroxide; Bandrowski's base, which is formed, is an insoluble colouring matter which can be eaten by experimental animals without ill effects, but apparently the phenylenediamine is not always completely oxidized, and to prevent the furs remaining toxic they are washed for from seven to eight hours; it is only when the washing has been scamped that the furs are toxic and cause dermatitis.

A similar process is used in hair dyeing; the hydrogen peroxide and the phenylenediamine are mixed, and the resulting solution is applied to the hair, but unfortunately a temptation exists not to wash too thoroughly as the washing may spoil the colour. Worse still, some who dye themselves apply the solutions separately, by which means they obtain a finer and more lasting colour, but attended probably by greater risk of a toxic result.

If careless men dye heavy moustaches in this manner, and women with similarly dyed hair hold tresses of it between their lips whilst dressing, the possibility of poisoning is increased. So long, therefore, as people are willing to use this dye in quantity, elderly-looking people with suspiciously dark brown hair may occasionally be met with whose paroxysmal tachycardia or indigestion, whose palpitation or liverishncss, or whose symptoms of gastric influenza will disappear if they will stop dyeing their hair.

But perhaps the most serious aspect of this case is the medico-legal one, for it cannot be doubted that this man was not far from death on the night he fell unconscious, and if he had been found dead, and an inquest had been held, would the true cause of death have been ascertained?

\section{TWO CASES OF ERYTHEMA NODOSUM WITH TUBERCULOUS BACILLAEMIA.} BY

JOHN A. WATT, M.B., B.CH., D.P.H.,

CLINICAL TUBERCULOSIS OFFICER, DERBYSHIRE COUNTY COUNCIL, AND

ERIC S. STUBBS, M.D., B.Sc., BELPER.

Professon Calmetre in his recent great work on Tuberculosis in Man and Animals affirms that erythema nodosum is caused by a bacillaemia. In 1913 Landouzy and Loederich, by grinding up and inoculating a nodule of erythema nodo-

* See Epitome (para. 61), British Medical Journal, January 19th, 1924. sum excised from a living patient succeeded in transmitting tuberculosis to a guinea-pig. This experimental proof was later corroborated by Hildebrandt, who infected a guineapig by inoculation with the fluid from a node. Much clinical evidence has accumulated in favour of the same view. Dr. H. J. Vetlesen, Christiania, has reviewed and summarized the literature and added a study of the records of his own hospital. ${ }^{1}$ H. Ernberg, at the first Northern Congress of Pediatrics in Copenhagen in 1919, reported an investigation into the fate of children who had suffered from erythe.na nodosum; of 35 who had so suffered between the years 1908 and 1914, he personally examined 31 during the winter of 1916-17. Not one had developed rheumatic fever or any sign of rheumatism, whereas in 13 cases symptoms of tuberculosis had supervened and one had died of miliary tuberculosis. In most the tuberculous symptoms had appeared during the first months or year after the outbreak of erythema nodosum. In a further clinical study of 55 cases he made an $x$-ray examination of the chest in 39, and found extensive tuberculous lesions due to active disease in 35 . The remaining 4 showed no radiographic changes in the lungs, but 2 had tuberculous glands of the neck and 2 subsequently developed phlyctenular conjurictivitis.

The two cases we report were striking because both occurred in a small mining village in Derbyshire within a few months of each other. Both showed extensive tuberculous infiltration of the lungs, and one has died of tuberculous meningitis. From Calmette's researches we know that tuberculous meningitis is never the result of a primary infection, but is a haematogenous infection nearly always caused by the pouring into the circulation of a quantity of bacilli through the rupture of caseated tubercles in the peribronchial or mediastinal lymph nodes in a subject recently and intensely infected, and who has not acquired any immunity against tuberculosis. The boy who died we know to have been in intimate daily personal contact with an aunt living in the adjoining house, who has since died from pulmonary tuberculosis. Both cases, too, had a long febrile illness marked by pallor, which could only be accounted for by an extcnsive infection.

$$
\text { CASE T. }
$$

A boy, aged 9, who previously had had good health, was taken ill at the beginning of February, 1923, and was found to be suffering from erythema nodosum. The rash appeared on the extensor aspect of both legs, the nodes being numerous and of moderate size (about a shilling). They underwent the usual changes of colour-red, livid, brownish-and as some faded fresh nodes appeared. The erythema was very persistent and lasted four or five weeks. The urine was free from albumin at this time. On February 24 th his temperature free from albumin at this time. On February 24 th his temperature was $101.8^{\circ}$, and it did not fall below $99^{\circ}$ until March 15th, only
becoming normal about April 25th. Early in April his urine was becoming normal about April 25th. Early in April his urine was Widal test was found to be negative. About April 22 nd he had pain with each breath on the right side of his chest. This lasted for about three days, but no pleural rub was heard. On May 11 th he was found to have distinct loss of resonance and defective air entry over the lower half of the right lung. A radiograph showed dense opacities in the lower half of the right lung and mottling of the opacities in the lower half of the right lung and mottling of the
upper half, radiating out from the hilus. The left base also showed upper half, radiating

From the beginning of his illness and for some time before the patient had complained of persistent headache, and it was feared tuberculous meningitis would develop. There was a temporary improvement during May, but in June his condition got worse. At first he was irritable, then he became drowsy. got worse. Atred on June 26th. On June 28 th he was admitted to the Derbyshire Royal Infirmary under Dr. Barber, who to the Derbyshire Royal Infirmary under Dr. Barber, who found him drowsy, but irritable when roused. The abdomen was
retracted and there was a well marked Kernig sign. The pulse retracted and there was a well marked Kernig sign. The pulse
rate during the first week varied between 72 and 96, and during the second week between 112 and 160 . There was a remittent temperature up to about $101^{\circ}$. The respirations were between 24 and 28 until the last two days. Lumbar puncture was performed three times, the fluid was clear under pressure, no organisms were found, but there was a fair number of cells present, a few of which were but there was a fair number of cells present, a few of which were
polymorphs. The boy died on July 11th. There was no postmortcm examination.

\section{CASE Ir.}

A boy, aged 13, residing in the same village as Case $i$, was seen first on August 10th, 1923, when he was said to have had a rash on his legs for about a fortnight and a cough for five weeks. His temperature was then $101.8^{\circ}$, pulse 96 . He had marked erythema nodosum and the rash had the same characters and distribution as in Case $i$, except that the duration was much shorter. The urine was normal. A faint systolic murmur was heard at the apex. On was normal. A faint systolic murmur was heard at the apex. On August 13th he was found to have impaired resonance over the
upper half of the left lung, harsh respiratory murmur, but "no moist sounds. A radiograph taken on the same date showed diffise opacity of the upper half of the left lung. By August 18th the 